\title{
On the measurement of the Hubble constant in a local low-density universe
}

\author{
Xiang-Ping $\mathrm{Wu}^{1}$, Zugan Deng ${ }^{1,2}$, Zhenlong Zou ${ }^{1}$, Li-Zhi Fang $^{3}$ \& Bo Qin ${ }^{1}$ \\ ${ }^{1}$ Beijing Astronomical Observatory, Chinese Academy of Sciences, Beijing 100080, China \\ ${ }^{2}$ Department of Physics, Graduate School, Chinese Academy of Sciences, Beijing 100039, China \\ ${ }^{3}$ Department of Physics and Steward Observatory, University of Arizona, Tucson, AZ 85721, USA
}

Submitted to

The Astrophysical Journal Letters

(Received )

E-mail: wxp@bao01.bao.ac.cn also wxp\%melamb@mesiob.obspm.fr 


\title{
ON THE MEASUREMENT OF THE HUBBLE CONSTANT IN A LOCAL LOW-DENSITY UNIVERSE
}

\author{
Xiang-Ping $\mathrm{Wu}^{1}$, Zugan Deng ${ }^{1,2}$, Zhenlong Zou ${ }^{1}$, Li-Zhi Fang ${ }^{3}$ \& Bo Qin ${ }^{4}$ \\ ${ }^{1}$ Beijing Astronomical Observatory, Chinese Academy of Sciences, Beijing 100080, China \\ ${ }^{2}$ Department of Physics, Graduate School, Chinese Academy of Sciences, Beijing 100039, China \\ ${ }^{3}$ Department of Physics and Steward Observatory, University of Arizona, Tucson, AZ 85721, USA
}

\begin{abstract}
Astrophysical observations indicate that the "Local Universe" has a relatively lower matter density $\left(\Omega_{0}\right)$ than the predictions of the standard inflation cosmology and the large-scale motions of galaxies which provide a mean mass density to be very close to unity. In such a local underdense region the Hubble expansion may not be representative of the global behaviour. Utilizing an underdense sphere embedded in a flat universe as the model of our "Local Universe", we show that the local Hubble constant would be $1.2-1.4$ times larger than the global value on scale of $\sim 80 \mathrm{Mpc}$, depending on the variation of $\Omega_{0}$. This may account for the recent measurements of the unpleasantly large Hubble constant of $\sim 80$ $\mathrm{km} / \mathrm{s} / \mathrm{Mpc}$ using the Cepheid variables in the Virgo cluster and the relative distance between Virgo and Coma cluster and removes the resulted apparent paradox of the age of our universe.
\end{abstract}

Subject headings: cosmology: distance scale - large-scale structure of universe

\section{INTRODUCTION}

It is firmly established from the dynamical analysis that the mean matter density of the Local Supercluster on scale of $\sim 10 \mathrm{Mpc}$ is within a factor of two of $\Omega_{0}=0.1$, which is about an order of magnitude smaller than the global value of $\Omega_{0}=1$ favoured by the inflation cosmological model. Indeed, the large-scale motions of galaxies with the assumption of "light-trace-mass" suggest an increasing tendency of $\Omega_{0}$ with scale up to $\sim 100$ Mpc [see Dekel (1994) for a recent review]. It is likely that we are situated in a local 
low-density "universe". Therefore, the local properties can significantly differ from the global ones and any local astrophysical measurements may not actually be representative of the real universe. Although this general argument is not entirely a new one [Readers are recommended to refer to Turner, Cen \& Ostriker (1992, references therein)], not much attention has been really concentrated on the issue, due to the unknown details of theoretical consideration on how large the differences between the local measurements and the global ones would be.

The critical question arises from the two recent independent measurements of the Hubble constant through the observations of Cepheid variables in the Virgo cluster (Pierce et al. 1994; Freedman et al. 1994), both of which give rise to a high value of $H_{0} \approx 80$ $\mathrm{km} / \mathrm{s} / \mathrm{Mpc}$. In the frame of standard cosmological model that assumes a completely homogeneous matter distribution everywhere in a flat universe, this large $H_{0}$ leads to a conflict that the expansion age of the Universe $(\sim 8 \mathrm{Gyr})$ is smaller than the age of globular clusters of the Galaxy ( $\sim 16 \mathrm{Gyr}$ ), implying that either the standard cosmological model needs to be revised or the present measurements of stellar ages need to be re-examined. This situation is indeed unfortunate as the standard Big Bang model and the theories of stellar evolution are two fundamentals of modern astrophysics and any modifications may have significant impacts on our conventional views. To avoid the difficulties resulted from the new measurements of $H_{0}$, we explore the possibility of attributing the high $H_{0}$ to a local low-density region which expands faster than the rest of the universe. This may survive the standard cosmological and stellar evolutionary theories.

\section{MODEL AND RESULTS}

The Tolman-Bondi metric is often used for the description of the space-time of an overdense or an underdense spherical region embedded in an expanding Universe (e.g. Zel'dovich \& Grishchuk 1984; Arnau et al. 1993; Fang \& Wu 1993; Wu \& Fang 1994):

$$
d s^{2}=\frac{r^{2}}{1-\epsilon f^{2}(x)} d x^{2}+r^{2}(x, t)\left(d \theta^{2}+\sin ^{2} \theta d \phi^{2}\right)-d t^{2} .
$$


The evolution of $r(x, t)$ is given by

$$
\dot{r}^{2}=-\epsilon f^{2}(x)+\frac{F(x)}{r}
$$

and $F(x)$ relates with the invariant mass $M(x)$ within $x$ through

$$
F(x)=2 M(x)=\int_{0}^{r} 8 \pi x^{2} \rho(x, t) d x \equiv \frac{8 \pi}{3} r_{i}^{3} \bar{\rho}_{i}
$$

where the prime denotes a derivative with respect to $x$, the dot with respect to $t, \rho$ and $\bar{\rho}$ are the matter density and the mean matter density, respectively. We use the subscript " $i$ " to stand for the "initial epoch" and "o" for the "present time".

Assuming a flat universe as the background, we can write the solution to an underdense region as $(\epsilon=-1)$

$$
\begin{aligned}
& \frac{S}{S_{i}}=\frac{1-\delta_{i}}{\delta_{i}} \frac{\cosh \eta-1}{2} \\
& \sinh \eta-\eta=\frac{4}{3} \frac{\delta_{i}^{3 / 2}}{1-\delta_{i}}\left(\frac{t}{t_{i}}-1\right)+\frac{2 \delta_{i}^{1 / 2}}{1-\delta_{i}}-\cosh ^{-1} \frac{1+\delta_{i}}{1-\delta_{i}} .
\end{aligned}
$$

Here $S$ is the expansion factor of the universe defined by $r=S(x, t) x, \delta_{i}$, the mean initial matter perturbation given by $\overline{\rho_{i}}=\rho_{c i}\left(1-\delta_{i}\right)$, and $\rho_{c}$, the critical mass density of the universe. The present Hubble constant is introduced through $H_{0}=\dot{S}_{0} / S_{0}$, which is generally a function of the radial coordinate $x$, i.e., the expansion rate may vary not only with time but also with position. We obtain the relation between the present local Hubble constant $\left(H_{L}\right)$ in the underdense region and the present global Hubble constant $\left(H_{G}\right)$ in the background universe to be

$$
\frac{H_{L}}{H_{G}}=\frac{\left(1+z_{i}\right)^{3 / 2}}{\left(S_{0} / S_{i}\right)}\left[\delta_{i}+\frac{1-\delta_{i}}{\left(S_{0} / S_{i}\right)}\right]^{1 / 2}
$$

in which $z_{i}$ is the redshift at the epoch $t=t_{i}$ when the initial density perturbation occurred. For simplicity, we choose $t_{i}$ to be the decoupling time that corresponds to $z_{i} \approx 1000$. The present density contrast $\frac{\Delta \rho}{\rho}$ stemming from the initial density perturbation profile $\delta(x)$ is found to be

$$
\frac{\Delta \rho}{\rho}=1-\left[\frac{1+z_{i}}{\left(S_{0} / S_{i}\right)}\right]^{3} \frac{1-\delta(x)}{1+d \ln S_{0} / d \ln x}
$$


and the present density parameter is $\Omega_{0}=1-\frac{\Delta \rho}{\rho}$.

We first adopt a constant density perturbation profile $\delta(x)=\delta_{i}=\delta_{0}$ in the local underdense region. In this case, the present Hubble constant inside the underdense sphere depends uniquely on the present matter density $\Omega_{0}$, as is shown in Figure 1 . In fact, this corresponds to an ensemble of solutions to the expanding "universe" with different $\Omega_{0}(<1)$. Utilizing this model to our local universe, a present matter density of $\Omega_{0}=0.2$ results in a local Hubble constant of 1.33 times larger than the global one and the $\Omega_{0}=0.5$ gives $H_{L}=1.19 H_{G}$. In the extreme case of $\Omega_{0}=0$, the local expansion rate is 1.5 times larger than that of the background universe of $\Omega_{0}=1$. Therefore, if we are unfortunately situated in a local low-density universe with a mass density of a few tenth of the critical value of the background universe, the local measurements like the two recent observations (Pierce et al. 1994; Freedman et al. 1994) utilizing the Cepheid variables of the Virgo cluster and the recession velocity of the Coma cluster would provide a relatively higher Hubble constant than the true value in the background flat universe.

Nevertheless, the local universe on scale of as large as the distance to Coma cluster cannot be well described by a constant matter perturbation. It appears that $\Omega_{0}$ varies from $\sim 0$ to $\sim 1$ with the increase of scale. We have then tested two initial density perturbation profiles that give rise to the similar shape of $\Omega_{0}$ to the observed one: $\delta(x)=\delta_{0} /\left[1+(x / a)^{2}\right]$ (the isothermal sphere with a core) and $\delta(x)=\delta_{0} /\left[1+(x / a)^{2}\right]^{3 / 2}$ (the King model), where $\delta_{0}$ determines the maximum initial density contrast and $a\left(a_{0}\right)$ is the initial (present) scale length of the perturbed region. Our computations show that these two profiles don't provide significantly different results of the present density contrast within $\sim 100 \mathrm{Mpc}$. Figure 2 demonstrates the variations of $\Delta \rho / \rho$ with distance for three sets of parameters in the King model. Although these curves might not exactly fit to the true distribution of $\Omega_{0}$ which has been unknown to date, they essentially represent the variation tendency of $\Omega_{0}$ with scale, which provide $\Omega_{0} \sim 0.1$ on scale of $10 \mathrm{Mpc}$ and $\Omega_{0} \sim(0.4-0.9)$ on scale of $100 \mathrm{Mpc}$. The Hubble constant variations with distance are shown in Figure 3 for the same parameters in Figure 2. At the distance of $\sim 80 \mathrm{Mpc}$ where the Coma cluster 
locates as were indicated by the recent observations, the local Hubble constant may be estimated to be 1.2 - 1.4 times larger than the global one, depending on the local matter content.

\section{DISCUSSION}

Recent measurements of the Hubble constant using the Cepheid variables in the Virgo cluster and the relative distance between the Virgo and the Coma cluster result in $H_{0}=87 \pm 7 \mathrm{~km} / \mathrm{s} / \mathrm{Mpc}$ (Pierce et al. 1994) and $H_{0}=80 \pm 17 \mathrm{~km} / \mathrm{s} / \mathrm{Mpc}$ (Freedman et al. 1994), which disagree with the other two HST measurements (Sandage et al. 1994; Saha et al. 1994) of $H_{0}=52 \pm 9 \mathrm{~km} / \mathrm{s} / \mathrm{Mpc}$ using the Cepheid variables and the brightness of the type Ia supernovae in two relatively closer galaxies (distance $=4.1-4.7$ $\mathrm{Mpc}$ ). The former determines actually the expansion rate of the Coma cluster which is so distant that the peculiar velocity contributes a negligible component and furthermore, the different methods produce the same mean relative Coma-Virgo distance modules. Whilst the later may suffer from the local calibration of the brightness of type Ia supernovae, leading to an overestimate of distance (Hogan 1994). We believe that the Coma recession velocity and the Coma-Virgo relative distance measurements are likely to reflect the nature of the local universe.

The apparent paradox of the "young" age of the universe may have arisen from the misuse of the local Hubble constant $H_{L}$ as the global value $H_{G}$. The Hubble constant of the background universe can be estimated by reducing the measured Hubble constant by a factor of $1.2-1.4$ at the distance of Coma cluster, leading to an increase of the currently estimated age of the universe by the same factor. Thus, the cosmological conflict between the expansion age of the universe, predicted in the standard cosmological model using the recent measurements of the large Hubble constant of $H_{0} \sim 80 \mathrm{~km} / \mathrm{s} / \mathrm{Mpc}$, and the ages of the oldest globular clusters of the Galaxy may vanish, or at least is partially resolved.

It appears that the true Hubble constant of the universe can be directly measured only 
when the observations are made beyond the local low-density region. It then remains to be promising that the time delay between the images of gravitationally lensed quasars at the redshift of $\sim 1$ may provide the reliable value of $H_{0}$. From the optical/radio monitoring of the double quasar $0957+561 \mathrm{~A}, \mathrm{~B}(z=1.41)$ over $\sim 10$-years coverage (Vanderriest et al. 1989; Roberts et al. 1991), which exhibits a time delay of 415/513 days, and the theoretical modeling of the lensing galaxies, one finds a Hubble constant of $H_{0}=48_{-7}^{+16}$ $/ 39_{-6}^{+13}$. Another evidence for supporting a low value of $H_{0} \sim 50 \mathrm{~km} / \mathrm{s} / \mathrm{Mpc}$ obtained in the distant universe comes from the measurement of the Sunyaev-Zel'dovich effect in Abell cluster A2218 ( $z=0.171)$ (Jones et al. 1993). Indeed, these two measurements of the Hubble constant at the cosmological distance yield a value apparently smaller than the local one, indicative of an expansion age of the universe comparable with the age of the oldest globular clusters. Bartlett et al (1994) have even claimed for a Hubble constant of as small as $30 \mathrm{~km} / \mathrm{s} / \mathrm{Mpc}$ and found that the small $H_{0}$ can overcome most of the difficulties in the current standard cosmological model. Further measurements of dynamical properties of the local universe and the future HST observations of the Cepheid variables in more distant galaxies are needed to confirm our arguments.

We thank Gary Mamon for reading the manuscript and for valuable comments. This work has been supported by the China National Science Foundation and the Chinese Academy of Sciences.

\section{References}

Arnau, J. V., Fullana, M. J., Monreal, L., \& Sáez, D. 1993, ApJ, 402, 359

Bartlett, J. G., Blanchard, A., Silk, J., \& Turner, M. S., 1994, Science, in press

Dekel, A. 1994, ARA\&A, 32, 371.

Freedman, W. L., et al. 1994, Nature, 371, 757

Fang, L. Z., \& Wu, X. P. 1993, ApJ, 408, 25

Hogan, C. J. 1994, Nature, 371, 374

Jones, M., et al. 1993, Nature, 365, 320 
Pierce, M. J., et al. 1994, Nature, 371, 385

Roberts, D. H., Lehár, J., Hewitt, J. N., \& Burke, B. F. 1991, Nature, 352, 43

Saha, A., et al. 1994, ApJ, 425, 14

Sandage, A. et al. 1994, ApJ, 423, L13

Turner, E. L., Cen R., \& Ostriker, J. P. 1992, AJ, 103, 1427

Vanderriest, C., et al. 1989, A\&A, 215, 1

Wu, X. P., \& Fang, L. Z. 1994, ApJ, 424, 530

Zel'dovich, Ya. B., \& Grishchuk, L. P. 1984, MNRAS, 207, 23p 


\section{Figure Captions}

Figure 1 The ratio of the local Hubble constant $H_{L}$ to the global value $H_{G}$ versus $\Omega_{0}$. The local underdense region is modelled by a sphere with constant matter density $\Omega_{0}$ embedded in a flat universe.

Figure 2 Variations of local matter density $\Omega_{0}$ with scale for the King model as the local negative density perturbations. Two parameters determine the model: the maximum initial density fluctuation $\delta_{0}$ and the present length scale $a_{0}$.

Figure 3 Variations of $H_{L} / H_{G}$ with scale for the three sets of parameters in Figure 2. 
This figure "fig1-1.png" is available in "png" format from: http://arxiv.org/ps/astro-ph/9412082v1 
This figure "fig1-2.png" is available in "png" format from: http://arxiv.org/ps/astro-ph/9412082v1 
This figure "fig1-3.png" is available in "png" format from: http://arxiv.org/ps/astro-ph/9412082v1 